\title{
Solving fuzzy Burgers equation by variational iteration method
}

\author{
Atimad Harir*, Said Melliani, L. Saadia Chadli \\ Laboratory of Applied Mathematics and Scientific Computing, Sultan Moulay Slimane University, P. O. Box 523, Beni Mellal, 23000, \\ Morocco.
}

\begin{abstract}
In this paper, the variational iteration method (VIM) has been applied to find the fuzzy solutions of the fuzzy Burgers equations with variable coefficients and fuzzy parameters. We follow the same strategy as in Buckley and Feuring which is: (1) first check to see if the Buckly-Feuring method produces a solution, and (2) if the Buckly-Feuring method does not give a solution, then see if the Seikkala procedure generates a solution. Several examples are given to show the new theorem of the Buckley-Feuring solution and the Seikkala solution.
\end{abstract}

Keywords: Fuzzy Burgers equations, variational iteration method, fuzzy number.

2020 MSC: 35R13, 46S40, 03E72.

(C)2020 All rights reserved.

\section{Introduction}

Zadeh is credited with introducing the concept of fuzzy sets in 1965 as a mathematical means of describing vagueness in linguistics. The idea may be considered as a generalization of classical set theory, in the decade since Zadeh's pioneering paper on fuzzy sets [22]. Assume Burgers models have imprecise parameters, since fuzzy sets theory is a powerful tool for modeling imprecise and processing vague in mathematical models, hence the our idea is solving Burgers equation with fuzzy parameters via the same strategy as Buckley and Feuring [4] using Variational Iteration Method (VIM) [13-15]. We begin this section with definition the notation we will use in the paper and then specify the fuzzy Burgers equation we wish to solve. In the next section we present the Buckly-Feuring solution and the Seikkala solution [18]. Also, in this section we give a sufficient condition for BF-solution to exist. The last section contains two examlpes where in the first example both solutions can exist in disjoint subregion of the domain as shown in Figs 1 and 2. In the last example the BF-solution does not exist but the S-solution can exist within some subregion of the domain as shown in Figs. 3, 4, and 5. In comparison with the paper [5, 17], we investigate nonlinear partial differential equations with fuzzy parameters, fuzzy initial value and fuzzy forcing functions, we propose a new theorem for finding the exact fuzzy solutions, witch extended to the Buckley-Feuring for the proposed models.

\footnotetext{
*Corresponding author

Email address: atimad harir@gmail . com (Atimad Harir)
}

doi: $10.22436 /$ jmcs.021.02.05

Received: 2019-05-16 Revised: 2019-09-07 Accepted: 2019-09-16 


\section{Preliminaries}

We place a bar over a capital letter to denote a fuzzy number of $\mathbb{R}^{n}$. So, $\bar{A}, \bar{K}, \bar{\varepsilon}, \bar{\beta}$ etc. all represent fuzzy numbers of $\mathbb{R}^{n}$ for some $n$. We write $\mu_{\bar{A}}(t)$, a number in $[0,1]$, for the membership function of $\bar{A}$ evaluated at $t \in \mathbb{R}^{n}$. An $\alpha$-cut of $\bar{A}$ is always a closed and bounded interval written as $\bar{A}[\alpha]$, is defined as $\left\{t \mid \mu_{\bar{A}}(t) \geqslant \alpha\right\}$ for $0<\alpha<1$. We separately specify $\bar{A}[0]$ as the closure of the union of all the $\bar{A}[\alpha]$ for $0<\alpha \leqslant 1$.

Definition 2.1 ([6]). Let $\mathbb{R}_{\mathcal{F}}=\{\bar{A} \mid \bar{A}: \mathbb{R} \rightarrow[0,1]$, satisfies (1)-(4) $\}$ :

(1) $\forall \bar{A} \in \mathbb{R}_{\mathcal{F}}, \bar{A}$ is normal;

(2) $\forall \bar{A} \in \mathbb{R}_{\mathcal{F}}, \bar{A}$ is a fuzzy convex set;

(3) $\forall \bar{A} \in \mathbb{R}_{\mathcal{F}}, \bar{A}$ is upper semi-continuous on $\mathbb{R}$;

(4) $\bar{A}[0]$ is a compact set.

Then $\mathbb{R}_{\mathcal{F}}$ is called fuzzy number space and $\forall \bar{A} \in \mathbb{R}_{\mathcal{F}}, \bar{A}$ is called a fuzzy number.

Definition $2.2([6,16])$. We represent an arbitrary fuzzy number by an ordered pair of functions $\bar{A}[\alpha]=$ $\left[A_{1}(\alpha), A_{2}(\alpha)\right], \alpha \in[0,1]$, which satisfy the following requirements:

1. $A_{1}(\alpha)$ is an increasing function over $[0,1]$;

2. $A_{2}(\alpha)$ is a decreasing function on $[0,1]$;

3. $A_{1}(\alpha)$ and $A_{2}(\alpha)$ are bounded left continuous on $(0,1]$, and right continuous at $\alpha=0$;

4. $A_{1}(\alpha) \leqslant A_{2}(\alpha)$, for $0 \leqslant \alpha \leqslant 1$.

Definition 2.3. For a fuzzy set $\bar{A}=\left(a_{1}, a_{2}, a_{3}\right),\left(a_{1}<a_{2}<a_{3}\right), \bar{A}$ is called triangular fuzzy number with peak $a_{2}$, left width $a_{2}-a_{1}>0$ and right width $a_{3}-a_{2}>0$, if its membership function has the following form:

$$
\mu_{\bar{A}}(t)= \begin{cases}1-\frac{\left(a_{2}-t\right)}{a_{2}-a_{1}}, & a_{1} \leqslant t \leqslant a_{2} \\ 1-\frac{\left(t-a_{2}\right)}{a_{3}-a_{2}}, & a_{2} \leqslant t \leqslant a_{3} \\ 0, & \text { otherwise. }\end{cases}
$$

Definition 2.4. For arbitrary fuzzy numbers $\bar{A}[\alpha]=\left[a_{1}(\alpha), a_{2}(\alpha)\right]$ and $\bar{B}[\alpha]=\left[b_{1}(\alpha), b_{2}(\alpha)\right]$ we have algebraic operations as follows:

1. $(\bar{A}+\bar{B})[\alpha]=\left[a_{1}(\alpha)+b_{1}(\alpha), a_{2}(\alpha)+b_{2}(\alpha)\right]$

2. $(\bar{A}-\bar{B})[\alpha]=\left[a_{1}(\alpha)-b_{2}(\alpha), a_{2}(\alpha)-b_{1}(\alpha)\right]$;

3. $k \bar{A}[\alpha]= \begin{cases}{\left[k a_{1}(\alpha), k a_{2}(\alpha)\right]} & k \geqslant 0, \\ {\left[k a_{2}(\alpha), k a_{1}(\alpha)\right]} & k<0 ;\end{cases}$

4. $(\bar{A} \cdot \bar{B})[\alpha]=\{\min z, \max z\}$ with $z=\left\{a_{1}(\alpha) \cdot b_{1}(\alpha), a_{1}(\alpha) \cdot b_{2}(\alpha), a_{2}(\alpha) \cdot b_{1}(\alpha), a_{2}(\alpha) \cdot b_{2}(\alpha)\right\}$;

5. If $0 \notin\left[b_{1}(\alpha), b_{2}(\alpha)\right], \frac{\bar{A}}{\bar{B}}[\alpha]=\left[\left(\frac{a_{1}}{b_{1}}\right)(\alpha),\left(\frac{a_{2}}{b_{2}}\right)(\alpha)\right]$, where

$$
\left(\frac{a_{1}}{b_{1}}\right)(\alpha)=\min \left\{\frac{a_{1}(\alpha)}{b_{1}(\alpha)}, \frac{a_{1}(\alpha)}{b_{2}(\alpha)}, \frac{a_{2}(\alpha)}{b_{1}(\alpha)}, \frac{a_{2}(\alpha)}{b_{2}(\alpha)}\right\}, \quad\left(\frac{a_{2}}{b_{2}}\right)(\alpha)=\max \left\{\frac{a_{1}(\alpha)}{b_{1}(\alpha)}, \frac{a_{1}(\alpha)}{b_{2}(\alpha)}, \frac{a_{2}(\alpha)}{b_{1}(\alpha)}, \frac{a_{2}(\alpha)}{b_{2}(\alpha)}\right\} .
$$

We adopt the general definition of a fuzzy number given in [7-9].

\section{Fuzzy Burgers equations}

Consider the one-dimensional Burgers equation has the form [1]

$$
\mathrm{U}_{\mathrm{t}}(\mathrm{t}, x)+\mathrm{P}(\mathrm{t}, \mathrm{x}, \varepsilon, \mathrm{U}(\mathrm{t}, x)) \mathrm{U}_{x}(\mathrm{t}, x)+\mathrm{Q}(\mathrm{x}, \mathrm{v}) \mathrm{U}_{x x}(\mathrm{t}, x)=\mathrm{F}(\mathrm{t}, x, \mathrm{k}),
$$

or 


$$
U_{t}(t, x)+P(t, x, \varepsilon, U(t, x)) U_{x}(t, x)=Q(x, v) U_{x x}(t, x)+F(t, x, k),
$$

subject to certain initial and boundary conditions.

These initial and boundary conditions, in state one-dimensional, can come in a variety of forms such as $\mathrm{U}(0, x)=\mathrm{c}_{1}$ or $\mathrm{U}(0, x)=\mathrm{g}_{1}\left(x, \mathrm{c}_{2}\right)$ or $\mathrm{U}\left(\mathrm{M}_{1}, x\right)=\mathrm{g}_{2}\left(x, \mathrm{c}_{3}, \mathrm{c}_{4}\right), \ldots$ In this work the method is applied for the Burgers equation (3.1). For Eq. (3.2), the same discussion can be made. In following lines, the components of (3.1) are enumerated:

- $I_{1}=\left[0, M_{1}\right]$ and $I_{2}=\left[M_{2}, M_{3}\right]$ are two intervals, which $M_{n_{1}}\left(n_{1}=2,3\right)$ is negative or positive and $M_{1}>0$;

- $F(t, x, k), U(t, x), P(t, x, \varepsilon, U(t, x))$ and $Q(x, v)$ will be continuous functions for $(t, x) \in \prod_{j=1}^{2} I_{j}$;

- $\mathrm{Q}(\mathrm{x}, v)$ has a finite number of roots each $x \in \mathrm{I}_{2}$;

- $k=\left(k_{1}, \ldots, k_{n}\right), c=\left(c_{1}, \ldots, c_{m}\right), \varepsilon=\left(\varepsilon_{1}, \ldots, \varepsilon_{s}\right)$, and $v=\left(v_{1}, \ldots, v_{e}\right)$ are vectors of constants with $k_{j} \in \mathrm{J}_{j}, c_{i} \in \mathrm{L}_{i}$ and $\varepsilon_{r} \in \mathrm{H}_{\mathrm{r}}$ and $v_{\mathrm{l}} \in \mathrm{D}_{\mathrm{l}}$.

Assume that Eq. (3.1) has a solution

$$
\mathrm{U}(\mathrm{t}, \mathrm{x})=\mathrm{G}(\mathrm{t}, \mathrm{x}, \mathrm{k}, \mathrm{c}, \varepsilon, v)
$$

for $G$ and

$$
G_{t}(t, x, k, c, \varepsilon, v)+P(\varepsilon, G(t, x, k, c, \varepsilon, v)) G_{x}(t, x, k, c, \varepsilon, v)+Q(x, v) G_{x x}(t, x, k, c, \varepsilon, v)
$$

are continuous with $(t, x) \in \prod_{j=1}^{2} I_{j}, k \in J=\prod_{j=1}^{n} J_{j}, c \in L=\prod_{i=1}^{m} L_{i}, \varepsilon \in H=\prod_{r=1}^{s} H_{r}$ and $v \in D=\prod_{l=1}^{e} D_{l}$.

Suppose the constants $k_{j}, c_{i}, \varepsilon_{r}$ and $v_{l}$ are imprecise in their values. We will model this uncertainty by substituting triangular fuzzy numbers for the $k_{j}, c_{i}, \varepsilon_{r}$ and $v_{l}$. If we fuzzify (3.1), then we obtain the fuzzy Burgers equation. Using the extension principle, we compute $\bar{F}, \bar{P}$, and $\bar{Q}$ from $F, P$, and $Q$, where $\overline{\mathrm{F}}(\mathrm{t}, \mathrm{x}, \overline{\mathrm{K}})$ has $\overline{\mathrm{K}}=\left(\overline{\mathrm{k}}_{1}, \ldots, \overline{\mathrm{k}}_{\mathrm{n}}\right)$ and $\overline{\mathrm{P}}(\bar{\varepsilon}, \overline{\mathrm{U}})$ has $\bar{\varepsilon}=\left(\bar{\varepsilon}_{1}, \ldots, \bar{\varepsilon}_{s}\right)$ and $\overline{\mathrm{Q}}(\overline{\mathrm{v}})$ a $\bar{v}=\left(\bar{v}_{1}, \ldots, \bar{v}_{e}\right)$ for $\mathrm{k}_{\mathrm{j}}, \varepsilon_{\mathrm{r}}$ and $\beta_{\mathrm{l}}$ triangular fuzzy numbers in $\mathrm{J}_{j}(0 \leqslant j \leqslant n), \mathrm{H}_{\mathrm{r}}(0 \leqslant r \leqslant s)$ and $\mathrm{D}_{l}(0 \leqslant l \leqslant e)$.

The function $U$ is changed to $\bar{U}$ where $\bar{U}: \prod_{j=1}^{2} I_{j} \rightarrow \mathbb{R}_{\mathcal{F}}$. That is, $\bar{U}(t, x)$ is a fuzzy function. The fuzzy Burgers equation is

$$
\overline{\mathrm{U}}_{\mathrm{t}}(\mathrm{t}, x)+\overline{\mathrm{P}}(\bar{\varepsilon}, \overline{\mathrm{U}}(\mathrm{t}, x)) \overline{\mathrm{U}}_{x}(\mathrm{t}, x)+\overline{\mathrm{Q}}(\overline{\mathrm{v}}) \overline{\mathrm{U}}_{x x}(\mathrm{t}, x)=\overline{\mathrm{F}}(\mathrm{t}, x, \overline{\mathrm{K}}),
$$

subject to certain initial and boundary conditions. The initial and boundary conditions can be of the form

$$
\overline{\mathrm{u}}(0, x)=\overline{\mathrm{C}}_{1} \text { or } \overline{\mathrm{u}}(0, x)=\overline{\mathrm{g}}_{1}\left(x, \overline{\mathrm{C}}_{2}\right) \text { or } \overline{\mathrm{u}}\left(\mathrm{M}_{1}, x\right)=\overline{\mathrm{g}}_{2}\left(x, \overline{\mathrm{C}}_{3}, \overline{\mathrm{C}}_{4}\right) \text {. }
$$

The $\bar{g}_{j}$ is the fuzzification $g_{i}$ via extension principle. Then, we wish to solve the problem given in (3.4). Finally, we fuzzify $G$ in (3.3).

Let $\bar{Z}(t, x)=\bar{G}(t, x, \bar{K}, \bar{C}, \bar{\varepsilon}, \bar{\beta})$ where $\bar{Z}$ is computed using the extension principal and is a fuzzy solution. In Section 5, we will discuss the concept solution with the same strategy as Buckley-Feuring for fuzzy Burgers equation.

Let

$$
\overline{\mathrm{K}}[\alpha]=\prod_{j=1}^{\mathrm{n}} \overline{\mathrm{K}}_{j}[\alpha], \bar{\varepsilon}[\alpha]=\prod_{\mathrm{r}=1}^{\mathrm{s}} \bar{\varepsilon}_{\mathrm{r}}[\alpha], \overline{\mathrm{C}}[\alpha]=\prod_{i=1}^{\mathrm{m}} \overline{\mathrm{C}}_{\mathrm{i}}[\alpha] \text {, and } \bar{v}[\alpha]=\prod_{l=1}^{\mathrm{e}} \overline{\mathrm{v}}_{\mathrm{l}}[\alpha] \text {. }
$$




\section{The variational iteration method}

To illustrate the basic concepts of VIM, consider the following general nonlinear partial differential equation:

$$
\left\{\begin{array}{l}
\operatorname{LU}(t, x)+\operatorname{RU}(t, x)+N U(t, x)=F(t, x, k), \\
U(t, 0)=g(t),
\end{array}\right.
$$

where $L=\frac{\partial}{\partial t}, R$ is a linear operator which has partial derivatives with respect to $x, N$ is a nonlinear operator, also $F(t, x, k)$ is the source non-homogeneous term. According to the VIM [20, 21], we can construct the following iteration formula:

$$
\mathrm{U}_{\mathrm{n}+1}(\mathrm{t}, x)=\mathrm{U}_{\mathrm{n}}(\mathrm{t}, \mathrm{x})+\int_{0}^{t} \lambda\left\{\mathrm{Lu}_{\mathrm{n}}+\mathrm{R} \widetilde{U}_{\mathrm{n}}+\mathrm{N} \widetilde{U}_{\mathrm{n}}-\mathrm{F}(\mathrm{s}, \mathrm{x}, \mathrm{k})\right\} \mathrm{ds},
$$

where $\lambda$ is general Lagrange multiplier [14], which can be identified optimally via the variational theory $[15,20], R \widetilde{U}_{n}$ and $N \widetilde{U}_{n}$ are considered as restricted variation, i.e. $\delta R \widetilde{U}_{n}=0, \delta N \widetilde{U}_{n}=0$. First, it is required to determine the Lagrange multipliers $\lambda$ that will be identified optimally via integration by parts. The approximations $u_{n+1}, n \geqslant 0$, calculate variation with respect to $u_{n}$, the following stationary conditions are obtained:

$$
\lambda^{\prime}(s)=0, \quad 1+\left.\lambda(s)\right|_{s=t}=0 .
$$

So, the Lagrange multiplier is $\lambda=-1$. Submitting the results into (4.1) leads to the following iteration formula

$$
\mathrm{U}_{\mathrm{n}+1}(\mathrm{t}, \mathrm{x})=\mathrm{U}_{\mathrm{n}}(\mathrm{t}, \mathrm{x})-\int_{0}^{t}\left\{\mathrm{LU}_{\mathrm{n}}+\mathrm{RU}_{\mathrm{n}}+\mathrm{N} \mathrm{U}_{\mathrm{n}}-\mathrm{F}(\mathrm{s}, \mathrm{x}, \mathrm{k})\right\} \mathrm{ds} .
$$

The second term on the right is called the correction term (4.2) can be solved iteratively using $\mathrm{U}_{0}(\mathrm{t}, \mathrm{x})=$ $g(x)$ as an initial approximation. Also the VIM used for system of linear and nonlinear partial differential equation in [20] can be handled to obtain Seikkala solution.

\section{Buckley-Feuring solution (BFS) and Seikkala solution (SS)}

Buckley-Feuring first presented the BFS in $[3,4]$. They define for all $t, x$ and $\alpha \in[0,1]$,

$$
\bar{Z}(t, x)[\alpha]=\left[z_{1}(t, x, \alpha), z_{2}(t, x, \alpha)\right], \quad \bar{F}(t, x, \bar{k})[\alpha]=\left[F_{1}(t, x, \alpha), F_{2}(t, x, \alpha)\right],
$$

and to check (3.4) we must compute $\bar{P}(t, x, \bar{\varepsilon}, \bar{Z})$ and $\bar{Q}(x, \bar{v})$.

Let $W=\overline{\mathrm{K}}[\alpha] \times \overline{\mathrm{C}}[\alpha] \times \bar{\varepsilon}[\alpha] \times \overline{\mathrm{v}}[\alpha]$. By definition

$$
\begin{aligned}
& z_{1}(t, x, \alpha)=\min \{G(t, x, k, c, \varepsilon, v):(k, c, \varepsilon, v) \in W\}, \\
& z_{2}(t, x, \alpha)=\max \{G(t, x, k, c, \varepsilon, v):(k, c, \varepsilon, v) \in W\}, \\
& F_{1}(t, x, \alpha)=\min \{F(t, x, k): k \in \bar{K}[\alpha]\}, \\
& F_{2}(t, x, \alpha)=\max \{F(t, x, k): k \in \bar{K}[\alpha]\},
\end{aligned}
$$

$\forall(t, x) \in \prod_{j=1}^{2} I_{j}$ and $\alpha \in[0,1]$. The $\alpha$-cuts of $\bar{Q}(x, \bar{v})$ can be found as follows: $\forall \alpha \in[0,1]$

$$
\overline{\mathrm{Q}}(x, \bar{v})[\alpha]=\left[\mathrm{Q}_{1}(x, \alpha), \mathrm{Q}_{2}(\mathrm{x}, \alpha)\right], \mathrm{Q}_{1}(x, \alpha)=\min \{\mathrm{Q}(x, v) \mid v \in \bar{v}[\alpha]\}, \mathrm{Q}_{2}(x, \alpha)=\max \{\mathrm{Q}(x, v) \mid v \in \bar{v}[\alpha]\},
$$


$\forall x \in I_{2}$ and $\alpha \in[0,1]$. Now for $\bar{Z}(t, x)$ to be a solution to the fuzzy initial value problem we need that $\frac{\partial}{\partial t} \bar{Z}(t, x), \frac{\partial}{\partial x} \bar{Z}(t, x)$ and $\frac{\partial^{2}}{\partial x^{2}} \bar{Z}(t, x)$ exist but also (3.4) must hold. To check (3.4) we must first compute $\bar{P}(t, x, \bar{\varepsilon}, \bar{Z}) . \alpha$-cuts of $\bar{P}(t, x, \bar{\varepsilon}, \bar{Z})$ can be found as follows:

$$
\bar{P}(t, x, \bar{\varepsilon}, \bar{Z})[\alpha]=\left[P_{1}(t, x, \alpha), P_{2}(t, x, \alpha)\right]
$$

with

$$
\begin{aligned}
& \mathrm{P}_{1}(\mathrm{t}, x, \alpha)=\min \{\mathrm{P}(\mathrm{t}, x, \varepsilon, z) \mid \varepsilon \in \bar{\varepsilon}[\alpha], z \in \overline{\mathrm{Z}}(\mathrm{t}, x)[\alpha]\}, \\
& \mathrm{P}_{2}(\mathrm{t}, x, \alpha)=\max \{\mathrm{P}(\mathrm{t}, x, \varepsilon, z) \mid \varepsilon \in \bar{\varepsilon}[\alpha], z \in \overline{\mathrm{Z}}(\mathrm{t}, x)[\alpha]\},
\end{aligned}
$$

$\forall(t, x) \in \prod_{j=1}^{2} I_{j}$ and $\alpha \in[0,1]$.

Assume that $\mathrm{P}(\mathrm{t}, \mathrm{x}, \varepsilon, z)>0, \mathrm{Q}(x, v)>0$ and the $z_{\mathfrak{i}}(\mathrm{t}, \mathrm{x}, \alpha) \mathfrak{i}=1,2$, has continuous partial derivatives so

$$
\left(z_{i}\right)_{t}+P_{i}(t, x, \alpha)\left(z_{i}\right)_{x}+Q_{i}(x, \alpha)\left(z_{i}\right)_{x x}
$$

is continuous for all $t, x \in \prod_{j=1}^{2} I_{j}$ and all $\alpha \in[0,1]$. Define

$$
\Gamma(t, x, \alpha)=\left[\left(z_{1}\right)_{t}+P_{1}(t, x, \alpha)\left(z_{1}\right)_{x}+Q_{1}(x, \alpha)\left(z_{1}\right)_{x \chi},\left(z_{2}\right)_{t}+P_{2}(t, x, \alpha)\left(z_{2}\right)_{x}+Q_{2}(x, \alpha)\left(z_{2}\right)_{x x}\right]
$$

for all $(t, x) \in \prod_{j=1}^{2} I_{j}$ and all $\alpha$. If for each fixed $(t, x) \in \prod_{j=1}^{2} I_{j}, \Gamma(t, x, \alpha)$ defines the $\alpha$-cut of a fuzzy number, then will be said that $\bar{Z}(t, x)$ is differentiable and is written as

$$
\bar{Z}_{t}[\alpha]+P(t, x, \bar{\varepsilon}, \bar{Z})[\alpha] \bar{Z}_{x}[\alpha]+Q(x, \bar{v})[\alpha] \bar{Z}_{x x}[\alpha]=\Gamma(t, x, \alpha)
$$

for all $(t, x) \in \prod_{j=1}^{2} I_{j}$ and all $\alpha$.

Sufficient conditions for $\Gamma(t, x, \alpha)$ to define $\alpha$-cut of a fuzzy number are [7]:

(i) $\left(z_{1}\right)_{t}(t, x, \alpha)+P_{1}(t, x, \alpha)\left(z_{1}\right)_{x}(t, x, \alpha)+Q_{1}(x, \alpha)\left(z_{1}\right)_{x x}(t, x, \alpha)$ is an increasing function of $\alpha$ for each $(t, x) \in \prod_{j=1}^{2} I_{j}$

(ii) $\left(z_{2}\right)_{t}(t, x, \alpha)+P_{2}(t, x, \alpha)\left(z_{2}\right)_{x}(t, x, \alpha)+Q_{2}(x, \alpha)\left(z_{2}\right)_{x x}(t, x, \alpha)$ is a decreasing function of $\alpha$ for each $(t, x) \in \prod_{j=1}^{2} I_{j} ;$ and

(iii) for $(t, x) \in \prod_{j=1}^{2} I_{j}$,

$$
\begin{aligned}
\left(z_{1}\right)_{\mathrm{t}}(\mathrm{t}, \mathrm{x}, 1) & +\mathrm{P}_{1}(\mathrm{t}, \mathrm{x}, 1)\left(z_{1}\right)_{\chi}(\mathrm{t}, \mathrm{x}, 1)+\mathrm{Q}_{1}(\mathrm{x}, 1)\left(z_{1}\right)_{x \chi}(\mathrm{t}, \mathrm{x}, 1) \\
& \leqslant\left(z_{2}\right)_{\mathrm{t}}(\mathrm{t}, \mathrm{x}, 1)+\mathrm{P}_{2}(\mathrm{t}, \mathrm{x}, 1)\left(z_{2}\right)_{\mathrm{x}}(\mathrm{t}, \mathrm{x}, 1)+\mathrm{Q}_{2}(\mathrm{x}, 1)\left(z_{2}\right)_{\mathrm{x}}(\mathrm{t}, \mathrm{x}, 1) .
\end{aligned}
$$

Now we can assume that the $z_{\mathfrak{i}}(t, x, \alpha)$ have continuous partial derivatives, so

$$
\left(z_{i}\right)_{t}+P_{i}(t, x, \alpha)\left(z_{i}\right)_{x}+Q_{i}(x, \alpha)\left(z_{i}\right)_{x x}
$$

is continuous on $\prod_{j=1}^{2} I_{j} \times[0,1]$ for $i=1,2$. Hence, if conditions (i)-(iii) above hold, $\bar{Z}(t, x)$ is differentiable.

$\bar{Z}(t, x)$ will be a BFS of the fuzzy Burgers equation if,

(a) $\bar{Z}(t, x)$ is differentiable;

(b) (3.4) holds for $\overline{\mathrm{U}}(\mathrm{t}, \mathrm{x})=\overline{\mathrm{Z}}(\mathrm{t}, \mathrm{x})$;

(c) $\bar{Z}(t, x)$ satisfies the initial and boundary conditions. 
Since there is no specified particular initial and boundary conditions, then only is checked if (3.4) holds.

We will only say that $\bar{Z}(t, x)$ is a BFS (without the initial and boundary conditions) if $\bar{Z}(t, x)$ is differentiable and

$$
(\bar{Z})_{t}+\bar{P}(t, x, \bar{\varepsilon}, \bar{Z})(\bar{Z})_{x}+\bar{Q}(x, \bar{v})(\bar{Z})_{x x}=\bar{F}(t, x, \bar{k})
$$

or the following equations must hold

$$
\begin{aligned}
& \left(z_{1}\right)_{t}+P_{1}(t, x, \alpha)\left(z_{1}\right)_{x}+Q_{1}(x, \alpha)\left(z_{1}\right)_{x x}=F_{1}(t, x, \alpha), \\
& \left(z_{2}\right)_{t}+P_{2}(t, x, \alpha)\left(z_{2}\right)_{x}+Q_{2}(x, \alpha)\left(z_{2}\right)_{x x}=F_{2}(t, x, \alpha),
\end{aligned}
$$

for all $(t, x) \in \prod_{j=1}^{2} I_{j}$ and all $\alpha \in[0,1]$. If $\bar{Z}(t, x)$ is a BFS and it satisfies the initial and boundary conditions we will say that $\bar{Z}(t, x)$ is a BFS satisfying the initial and boundary conditions. If $\bar{Z}(t, x)$ is not a BFS, then we will consider the SS. At the end of this section we will present a sufficient condition for the existence of the BFS, but now let us define the SS [18]. Let

$$
\overline{\mathrm{u}}(\mathrm{t}, x)[\alpha]=\left[\mathrm{u}_{1}(\mathrm{t}, \mathrm{x}, \alpha), \mathrm{u}_{2}(\mathrm{t}, \mathrm{x}, \alpha)\right] .
$$

Consider the system of Burgers equations

$$
\begin{aligned}
& \left(u_{1}\right)_{t}+P_{1}(t, x, \alpha)\left(u_{1}\right)_{x}+Q_{1}(x, \alpha)\left(u_{1}\right)_{x x}=F_{1}(t, x, \alpha), \\
& \left(u_{2}\right)_{t}+P_{2}(t, x, \alpha)\left(u_{2}\right)_{x}+Q_{2}(x, \alpha)\left(u_{2}\right)_{x x}=F_{2}(t, x, \alpha),
\end{aligned}
$$

for all $(t, x) \in \prod_{j=1}^{2} I_{j}$ and $\alpha \in[0,1]$. We append to Eqs. (5.9) and (5.10) any initial and boundary conditions. For example, if they were $\overline{\mathrm{U}}(0, x)=\overline{\mathrm{C}}$, then we add

$$
u_{1}(0, x, \alpha)=c_{1}(\alpha), \quad u_{2}(0, x, \alpha)=c_{2}(\alpha),
$$

where $\bar{C}[\alpha]=\left[c_{1}(\alpha), c_{2}(\alpha)\right]$. Let $u_{i}(t, x, \alpha) i=1,2$ solve Eqs. (5.9) and (5.10) plus initial and boundary conditions. If

$$
\left[u_{1}(t, x, \alpha), u_{2}(t, x, \alpha)\right]
$$

defines the $\alpha$-cut of a fuzzy number for all $(t, x) \in \prod_{j=1}^{2} I_{j}$, then $\bar{U}(t, x)$ is the SS. Clearly if the BFS satisfying the boundary conditions is $\bar{Z}(t, x)$, then $\bar{Z}(t, x)$ is also the SS, see [5, 17, Theorem 5.2]. As we shall see the SS can exist when the BFS fails to exist.

Now we will present a sufficient condition for the BFS to exist such as Buckley and Feuring. Since there are such a variety of possible initial and boundary conditions, so we will omit them from the following theorem. One must separately check out the initial and boundary conditions. So, we will omit the constants $c_{i}, 1 \leqslant i \leqslant m$, from the problem. Therefore, (3.3) becomes $U(t, x)=G(t, x, k, \varepsilon, v)$, so

$$
\overline{\mathrm{Z}}(\mathrm{t}, \mathrm{x})=\overline{\mathrm{G}}(\mathrm{t}, \mathrm{x}, \overline{\mathrm{K}}, \bar{\varepsilon}, \overline{\mathrm{v}}) .
$$

Theorem 5.1. Assume $\bar{Z}(t, x)$ is differentiable.

(a) if

$$
\mathrm{P}\left(\mathrm{t}, x, \varepsilon_{i}, z\right)>0, \frac{\partial \mathrm{P}}{\partial z}>0 \text { and } \frac{\partial \mathrm{P}}{\partial \varepsilon_{i}} \frac{\partial \mathrm{G}}{\partial \varepsilon_{i}}>0, \quad \mathrm{t}, x \in \mathrm{I}_{1} \times \mathrm{I}_{2} \text { for } i=1,2, \ldots, \mathrm{m}
$$

and if

$$
\mathrm{Q}\left(x, v_{\mathrm{l}}\right)>0 \text { and } \frac{\partial \mathrm{Q}}{\partial v_{\mathrm{l}}} \frac{\partial \mathrm{G}}{\partial v_{\mathrm{l}}}>0, \quad x \in \mathrm{I}_{2} \text { for } \mathrm{l}=1,2, \ldots, \mathrm{e},
$$

and if

$$
\frac{\partial G}{\partial k_{j}} \frac{\partial F}{\partial k_{j}}>0 \text { for } j=1,2, \ldots, n,
$$

then BFS $=\bar{Z}(t, x)$. 
(b) If relations (5.11) does not hold for some i or relation (5.12) does not hold for some l, or relation (5.13) does not hold for some $\mathrm{j}$, then $\overline{\mathrm{Z}}(\mathrm{t}, \mathrm{x})$ is not a BFS.

Proof.

(a) For simplicity assume $k_{j}=k, \varepsilon_{i}=\varepsilon, v_{l}=v$ and $\frac{\partial G}{\partial k}<0, \frac{\partial F}{\partial k}<0, \frac{\partial P}{\partial \varepsilon}>0, \frac{\partial G}{\partial \varepsilon}>0, \frac{\partial Q}{\partial v}<0$ and $\frac{\partial G}{\partial v}<0$. The proof for $\frac{\partial G}{\partial k}>0, \frac{\partial F}{\partial k}>0, \frac{\partial P}{\partial \varepsilon}<0, \frac{\partial G}{\partial \varepsilon}<0, \frac{\partial Q}{\partial v}>0$ and $\frac{\partial G}{\partial v}>0$ is similar and omitted. Since $\frac{\partial G}{\partial k}<0, \frac{\partial G}{\partial \varepsilon}>0$ and $\frac{\partial G}{\partial v}<0$, then from (5.1) and (5.2) we have

$$
z_{1}(t, x, \alpha)=G\left(t, x, k_{2}(\alpha), \varepsilon_{1}(\alpha), v_{2}(\alpha)\right), \quad z_{2}(t, x, \alpha)=G\left(t, x, k_{1}(\alpha), \varepsilon_{2}(\alpha), v_{1}(\alpha)\right),
$$

from (5.3), (5.4), and $\frac{\partial F}{\partial k}<0$ we have

$$
F_{1}(t, x, \alpha)=F\left(t, x, k_{2}(\alpha)\right), \quad F_{2}(t, x, \alpha)=F\left(t, x, k_{1}(\alpha)\right),
$$

since (5.6), $\frac{\partial P}{\partial \varepsilon}>0$ and $\frac{\partial P}{\partial z}>0$ we have

$$
P_{1}(t, x, \alpha)=P\left(x, \varepsilon_{1}(\alpha), z_{1}(t, x, \alpha)\right), \quad P_{2}(t, x, \alpha)=P\left(x, \varepsilon_{2}(\alpha), z_{2}(t, x, \alpha)\right),
$$

from (5.5) and $\frac{\partial Q}{\partial v}<0$ we have

$$
\mathrm{Q}_{1}(x, \alpha)=\mathrm{Q}\left(x, v_{2}(\alpha)\right), \quad \mathrm{Q}_{2}(x, \alpha)=\mathrm{Q}\left(x, v_{1}(\alpha)\right),
$$

for all $\alpha \in[0,1]$, where

$$
\overline{\mathrm{K}}[\alpha]=\left[\mathrm{k}_{1}(\alpha), \mathrm{k}_{2}(\alpha)\right], \bar{\varepsilon}[\alpha]=\left[\varepsilon_{1}(\alpha), \varepsilon_{2}(\alpha)\right], \text { and } \bar{v}[\alpha]=\left[v_{1}(\alpha), v_{2}(\alpha)\right] .
$$

Now $G(t, x, k, \varepsilon, v)$ solves (3.2), which means

$$
G_{t}+P(t, x, \varepsilon, G) G_{x}+Q(x, v) G_{x x}=F(t, x, k)
$$

for all $(t, x) \in \prod_{j=1}^{2} I_{j}, k \in J, \varepsilon \in H$ and $v \in D$.

Suppose $\bar{Z}(t, x)$ is differentiable and $P(t, x, \varepsilon, z)>0$ and $Q(x, v)>0$, so

$$
\begin{aligned}
& \partial_{t} z_{1}(t, x, \alpha)+P_{1}(t, x, \alpha) \partial_{x} z_{1}(t, x, \alpha)+Q_{1}(x, \alpha) \partial_{x x} z_{1}(t, x, \alpha)=F_{1}(t, x, \alpha), \\
& \partial_{t} z_{2}(t, x, \alpha)+P_{2}(t, x, \alpha) \partial_{x} z_{2}(t, x, \alpha)+Q_{2}(x, \alpha) \partial_{x x} z_{2}(t, x, \alpha)=F_{2}(t, x, \alpha),
\end{aligned}
$$

for all $(t, x) \in \prod_{j=1}^{2} I_{j}$ and $\alpha \in[0,1]$.

Hence, (5.7) and (5.8) hold and $\bar{Z}(t, x)$ is a BFS.

(b) Now consider the situation where (5.11) or (5.12) or (5.13) does not hold.

Let us only look at one case where $\frac{\partial Q}{\partial v}<0$ (assume $\frac{\partial G}{\partial k}>0, \frac{\partial F}{\partial k}>0, \frac{\partial G}{\partial \varepsilon}>0, \frac{\partial P}{\partial \varepsilon}>0, \frac{\partial P}{\partial z}>0$ and $\frac{\partial G}{\partial v}>0$, $P(x, \varepsilon, z)>0$ and $Q(x, v)>0)$. Then we have

$$
\begin{aligned}
z_{1}(t, x, \alpha) & =G\left(t, x, k_{1}(\alpha), \varepsilon_{1}(\alpha), v_{1}(\alpha)\right) \\
z_{2}(t, x, \alpha) & =G\left(t, x, k_{2}(\alpha), \varepsilon_{2}(\alpha), v_{2}(\alpha)\right) \\
F_{1}(t, x, \alpha) & =F\left(t, x, k_{1}(\alpha)\right), \quad F_{2}(t, x, \alpha)=F\left(t, x, k_{2}(\alpha)\right), \\
P_{1}(t, x, \alpha) & =P\left(t, x, \varepsilon_{1}(\alpha), z_{1}(t, x, \alpha)\right), \quad P_{2}(t, x, \alpha)=P\left(t, x, \varepsilon_{2}(\alpha), z_{2}(t, x, \alpha)\right), \\
Q_{1}(x, \alpha) & =Q\left(x, v_{2}(\alpha)\right), \quad Q_{2}(x, \alpha)=Q\left(x, v_{1}(\alpha)\right),
\end{aligned}
$$


then we have

$$
\begin{aligned}
& \partial_{t} z_{1}(t, x, \alpha)+P_{1}(t, x, \alpha) \partial_{x} z_{1}(t, x, \alpha)+Q_{1}(x, \alpha) \partial_{x x} z_{1}(t, x, \alpha)=F_{1}(t, x, \alpha), \\
& \partial_{t} z_{2}(t, x, \alpha)+P_{2}(t, x, \alpha) \partial_{x} z_{2}(t, x, \alpha)+Q_{2}(x, \alpha) \partial_{x x} z_{2}(t, x, \alpha)=F_{2}(t, x, \alpha),
\end{aligned}
$$

which is not true, because

$$
\begin{aligned}
& G_{t}\left(t, x, k_{1}(\alpha), \varepsilon_{1}(\alpha), v_{1}(\alpha)\right)+P\left(t, x, \varepsilon_{1}(\alpha), G\left(t, x, k_{1}(\alpha), \varepsilon_{1}(\alpha), v_{1}(\alpha)\right)\right) G_{x}\left(t, x, k_{1}(\alpha), \varepsilon_{1}(\alpha), v_{1}(\alpha)\right) \\
& \quad+Q\left(x, v_{2}(\alpha)\right) G_{x x}\left(t, x, k_{1}(\alpha), \varepsilon_{1}(\alpha), v_{1}(\alpha)\right)=F\left(t, x, k_{1}(\alpha)\right), \\
& G_{t}\left(t, x, k_{2}(\alpha), \varepsilon_{2}(\alpha), v_{2}(\alpha)\right)+P\left(t, x, \varepsilon_{1}(\alpha), G\left(t, x, k_{1}(\alpha), \varepsilon_{2}(\alpha), v_{2}(\alpha)\right)\right) G_{x x}\left(t, x, k_{2}(\alpha), \varepsilon_{2}(\alpha), v_{2}(\alpha)\right) \\
& \quad+Q\left(x, v_{1}(\alpha)\right) G_{x x}\left(t, x, k_{1}(\alpha), \varepsilon_{1}(\alpha), v_{2}(\alpha)\right)=F\left(t, x, k_{2}(\alpha)\right) .
\end{aligned}
$$

Lemma 5.2. Assume $\bar{Z}(t, x)$ is differentiable.

(a) if

$$
\mathrm{P}\left(\mathrm{t}, x, \varepsilon_{i}, z\right)>0, \frac{\partial \mathrm{P}}{\partial z}>0, \text { and } \frac{\partial \mathrm{P}}{\partial \varepsilon_{i}} \frac{\partial \mathrm{G}}{\partial \varepsilon_{i}}>0, \quad \mathrm{t}, x \in \mathrm{I}_{1} \times \mathrm{I}_{2} \text { for } i=1,2, \ldots, \mathrm{m},
$$

and if

$$
\frac{\partial G}{\partial k_{j}} \frac{\partial F}{\partial k_{j}}>0, \text { for } j=1,2, \ldots, n,
$$

then $B F S=\bar{Z}(t, x)$.

(b) If relations (5.14) does not hold for some $i$, or relation (5.15) does not hold for some $j$, then $\bar{Z}(t, x)$ is not a BFS.

\section{Examples}

We consider the following examples from $[1,2,19]$ and we added fuzzy parameters.

Example 6.1. We first consider the one-dimensional Burgers equation with variable coefficients as

$$
\mathrm{U}_{\mathrm{t}}(\mathrm{t}, x)+\varepsilon \mathrm{U}(\mathrm{t}, \mathrm{x}) \mathrm{U}_{\mathrm{x}}(\mathrm{t}, \mathrm{x})=v \mathrm{U}_{\mathrm{xx}}(\mathrm{t}, \mathrm{x})+\mathrm{k}
$$

with the initial conditions

$$
u(0, x)=\frac{c}{2}\left(1-\tanh \left[\frac{c x}{4}\right]\right)
$$

where $\varepsilon=v x \in[0,1], \mathrm{t}>0, \mathrm{k} \in] 0, \mathrm{~J}], \varepsilon \in] 0,1]$ and $\mathrm{c} \in[0, \mathrm{~L}[$ are constants.

To solve Eq (6.1) by means of the VIM, substitute in Eq. (4.2) by

$$
R U_{n}=-\varepsilon\left(U_{n}\right)_{x x}, \quad N U_{n}=\varepsilon U_{n}\left(U_{n}\right)_{x},
$$

and $f(t, x, k)=k$ and obtain the following variational iteration formula:

$$
U_{n+1}(t, x)=U_{n}(t, x)-\int_{0}^{t}\left\{\left(U_{n}(s, x)\right)_{s}+\varepsilon U_{n}(s, x)\left(U_{n}(s, x)\right)_{x}-\varepsilon\left(U_{n}(s, x)\right)_{x x}-k\right\} d s .
$$

Using (6.2), the approximate solutions $U_{n}(t, x)$ are obtained iteratively by substituting:

$$
\mathrm{u}_{0}(\mathrm{t}, \mathrm{x})=\mathrm{u}(0, x)=\frac{\mathrm{c}}{2}\left(1-\tanh \left[\mathrm{c} \frac{\mathrm{x}}{4}\right]\right)
$$




$$
\begin{aligned}
& \mathrm{U}_{1}(\mathrm{t}, x)=\mathrm{U}_{0}(\mathrm{t}, \mathrm{x})+\varepsilon \frac{\mathrm{c}^{3}}{16} \operatorname{sech}^{2}\left[\mathrm{c} \frac{x}{4}\right] \mathrm{t}+\mathrm{kt}, \\
& \mathrm{U}_{2}(\mathrm{t}, \mathrm{x})=\mathrm{U}_{1}(\mathrm{t}, \mathrm{x})+\varepsilon^{2} \frac{\mathrm{c}^{5}}{128} \tanh \left[\mathrm{c} \frac{\mathrm{x}}{4}\right] \operatorname{sech}^{2}\left[\mathrm{c} \frac{\mathrm{x}}{4}\right] \mathrm{t}^{2}+\varepsilon k \frac{\mathrm{c}^{2}}{16} \operatorname{sech}^{2}\left[\mathrm{c} \frac{\mathrm{x}}{4}\right] \mathrm{t}^{2}+\varepsilon^{3} \frac{\mathrm{c}^{7}}{1536} \tanh \left[\mathrm{c} \frac{\mathrm{x}}{4}\right] \operatorname{sech}^{4}\left[\mathrm{c} \frac{x}{4}\right] \mathrm{t}^{3},
\end{aligned}
$$

and so on. The approximate solution takes the form $U(t, x) \simeq U_{n}(t, x)$, where $n$ is the final step, which gives the exact solution of Eq. (6.1) takes the form

$$
\mathrm{U}(\mathrm{t}, \mathrm{x})=\frac{\mathrm{c}}{2}\left(1-\tanh \left[\frac{\mathrm{c}}{4}\left(\mathrm{x}-\left(\frac{\varepsilon}{2}\right)\left(\mathrm{ct}+\mathrm{kt} \mathrm{t}^{2}\right)\right)\right]\right)+\mathrm{kt} .
$$

Now we fuzzify $F(t, x, k), Q(x, \varepsilon)$, and $P(t, x, \varepsilon, G)$,

$$
\mathrm{G}(\mathrm{t}, x, k, c, \varepsilon)=\frac{\mathrm{c}}{2}\left(1-\tanh \left[\frac{\mathrm{c}}{4}\left(x-\left(\frac{\varepsilon}{2}\right)\left(\mathrm{ct}+k \mathrm{t}^{2}\right)\right)\right]\right)+\mathrm{kt} .
$$

Clearly

$$
\overline{\mathrm{F}}(\mathrm{t}, \mathrm{x}, \overline{\mathrm{K}})=\overline{\mathrm{K}}, \quad \overline{\mathrm{Q}}(\mathrm{x}, \bar{\varepsilon})=\bar{\varepsilon},
$$

so that

$$
F_{1}(t, x, \alpha)=k_{1}(\alpha), \quad F_{2}(t, x, \alpha)=k_{2}(\alpha), \quad Q_{1}(x, \alpha)=\varepsilon_{1}(\alpha), \quad Q_{2}(x, \alpha)=\varepsilon_{2}(\alpha) .
$$

Also

$$
\overline{\mathrm{G}}(\mathrm{t}, x, \overline{\mathrm{K}}, \overline{\mathrm{C}}, \bar{\varepsilon})=\frac{\overline{\mathrm{C}}}{2}\left(1-\tanh \left[\frac{\overline{\mathrm{C}}}{4}\left(x-\left(\frac{\bar{\varepsilon}}{2}\right)\left(\overline{\mathrm{C}} \mathrm{t}+\overline{\mathrm{K}} \mathrm{t}^{2}\right)\right)\right]\right)+\overline{\mathrm{K}} \mathrm{t}
$$

therefore

$$
z_{i}(t, x, \alpha)=\frac{c_{i}}{2}\left(1-\tanh \left[\frac{c_{i}}{4}\left(x-\left(\frac{\varepsilon_{i}}{2}\right)\left(c_{i} t+k_{i} t^{2}\right)\right)\right]\right)+k_{i} t,
$$

for $i=1,2$ and $\bar{C}>0$, i.e., $\left(\bar{C}=\left(c_{1}, c_{2}, c_{3}\right)\right.$ also with $\left.c_{1}>0\right), \bar{K}[\alpha]=\left[k_{1}(\alpha), k_{2}(\alpha)\right], \bar{C}[\alpha]=\left[c_{1}(\alpha), c_{2}(\alpha)\right]$, and $\bar{\varepsilon}[\alpha]=\left[\varepsilon_{1}(\alpha), \varepsilon_{2}(\alpha)\right]$.

$\bar{Z}(t, x)$ is differentiable because $\left(z_{\mathfrak{i}}(t, x, \alpha)\right)_{\mathfrak{t}}+\varepsilon_{\mathfrak{i}}(\alpha) z_{\mathfrak{i}}(t, x, \alpha)\left(z_{\mathfrak{i}}(t, x, \alpha)\right)_{x}-\varepsilon_{\mathfrak{i}}(\alpha)\left(z_{\mathfrak{i}}(t, x, \alpha)\right)_{x x}$ for $\mathfrak{i}=$ 1,2 are $\alpha$-cuts of $\bar{K}$, i.e., $\alpha$-cuts of a fuzzy number. Due to

$$
\begin{aligned}
P(t, x, \varepsilon, z) & >0, \quad \frac{\partial P}{\partial z}>0, \\
\frac{\partial G}{\partial k} & =\frac{c^{2}}{16} \varepsilon \operatorname{sech}^{2}\left(\frac{c}{4}\left(x-\frac{\varepsilon}{2}\left(c t+k t^{2}\right)\right)\right) t^{2}+t>0, \quad \frac{\partial F}{\partial k}>0, \\
\frac{\partial P}{\partial \varepsilon} & =z>0, \quad \frac{\partial G}{\partial \varepsilon}=\frac{c^{2}}{16} \operatorname{sech}^{2}\left(\frac{c}{4}\left(x-\frac{\varepsilon}{2}\left(c t+k t^{2}\right)\right)\right)\left(c t+k t^{2}\right)>0 .
\end{aligned}
$$

That is, $(\bar{Z})_{t}+\bar{\varepsilon} \bar{Z}(\bar{Z})_{x}=\bar{\varepsilon}(\bar{Z})_{x x}+\bar{K}$, a fuzzy number. So Theorem 5.1 implies the result that $\bar{Z}(t, x)$ is a BFS, see Fig. 1, 2, and Table 1 . We easily see that

$$
z_{\mathfrak{i}}(0, x, \alpha)=\frac{c_{i}}{2}\left(1-\tanh \left[c_{i} \frac{x}{4}\right]\right)
$$

for $i=1,2$, so $\bar{Z}(t, x)$ also satisfies the initial condition. The BFS that satisfies the initial condition may be written as

$$
\overline{\mathrm{Z}}(\mathrm{t}, \mathrm{x})=\frac{\overline{\mathrm{C}}}{2}\left(1-\tanh \left[\frac{\overline{\mathrm{C}}}{4}\left(x-\left(\frac{\bar{\varepsilon}}{2}\right)\left(\overline{\mathrm{C}} \mathrm{t}+\overline{\mathrm{K}} \mathrm{t}^{2}\right)\right)\right]\right)+\overline{\mathrm{K}} \mathrm{t}
$$

for all $\mathrm{t}>0, x \in[0,1]$. 
Table 1: Comparison of the solution $u(t, x)$ with $u_{1}(t, x, \alpha)$ and $u_{2}(t, x, \alpha)$ for $\alpha=0, \bar{\varepsilon}=\overline{1}, \bar{c}=\overline{4}$ and $\bar{k}=\overline{2}$.

\begin{tabular}{|c|c|c|c|c|c|c|c|c|c|}
\hline & \multicolumn{3}{|c|}{$u(t, x)$} & \multicolumn{3}{c|}{$\mathfrak{u}_{1}(t, x, \alpha)$} & \multicolumn{3}{c|}{$\mathfrak{u}_{2}(t, x, \alpha)$} \\
\hline$t \backslash x$ & 0.0099 & 0.0198 & 0.0297 & 0.0099 & 0.0198 & 0.0297 & 0.0099 & 0.0198 & 0.0297 \\
\hline 0 & 2.1196 & 2.2404 & 2.3623 & 1.5198 & 1.5396 & 1.5594 & 2.8709 & 3.2391 & 3.5948 \\
0.0198 & 2.0998 & 2.207 & 2.3427 & 1.5087 & 1.5285 & 1.5483 & 2.8404 & 3.2100 & 3.5679 \\
0.0396 & 2.080 & 2.201 & 2.3232 & 1.4975 & 1.5173 & 1.5371 & 2.8098 & 3.1512 & 3.5407 \\
\hline
\end{tabular}

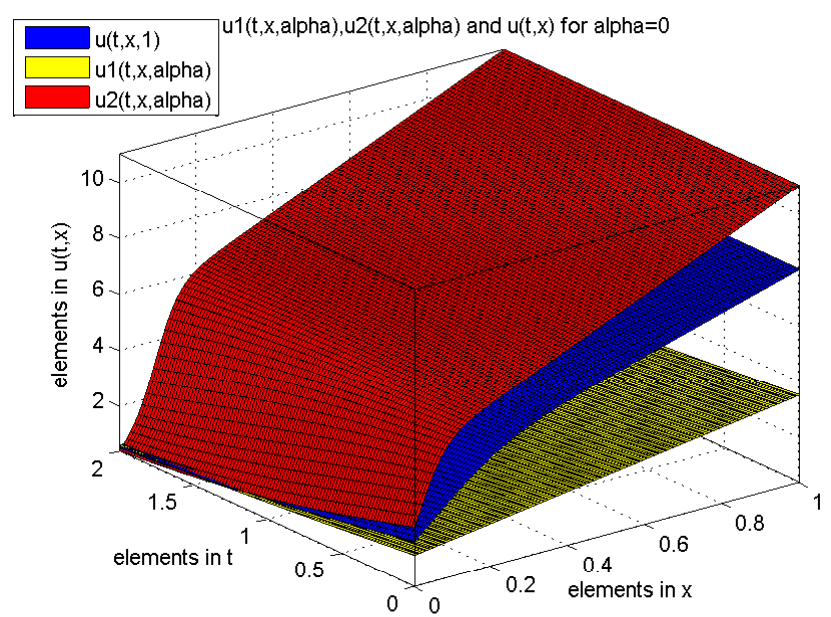

Figure 1: The surfaces shows the fuzzy solution for $\alpha=0$ for (6.1), when $\bar{c}=\overline{4}, \bar{k}=\overline{2}$ and $\bar{\varepsilon}=\overline{1}$.

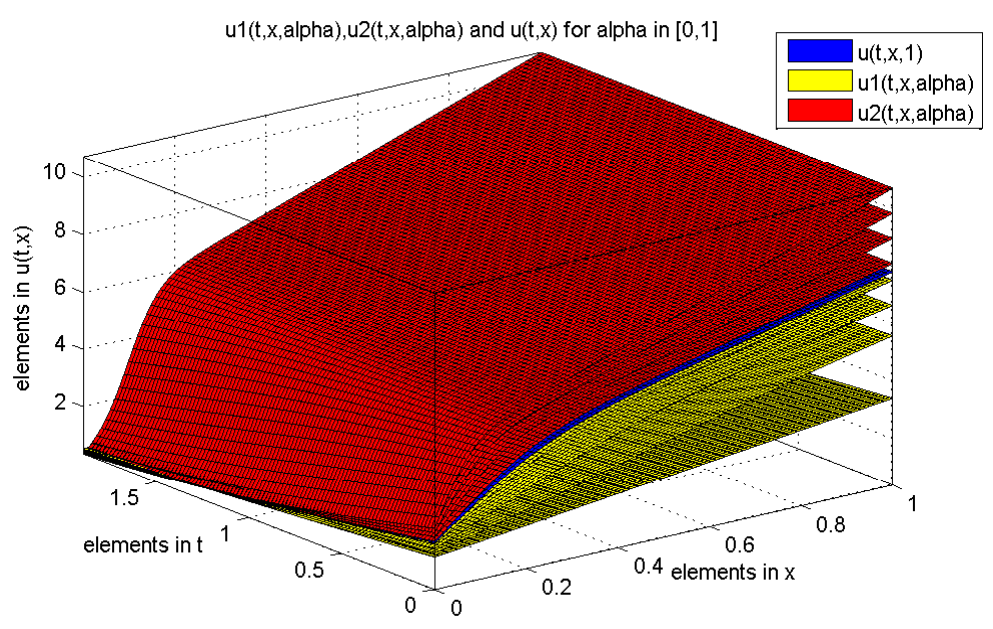

Figure 2: The surfaces shows the fuzzy solution for $\alpha \in[0,1]$ for $(6.1)$, when $\bar{c}=\overline{4}, \bar{k}=\overline{2}$ and $\bar{\varepsilon}=\overline{1}$.

Example 6.2. We consider a model partial differential equation described by

$$
U_{t}(t, x)-\varepsilon U(t, x) U_{x}(t, x)=2 k
$$

with the initial conditions

$$
\mathrm{u}(0, x)=\frac{x}{2 \varepsilon},
$$

where $x \in[0,1], t>0, k \in] 0,2], \varepsilon \in[-2,0[$ are constants. 
Similarly we can establish an iteration formula in the form

$$
u_{n+1}(t, x)=u_{n}(t, x)-\int_{0}^{t}\left\{\left(u_{n}(s, x)\right)_{s}-\varepsilon u_{n}(s, x)\left(U_{n}(s, x)\right)_{x}-2 k\right\} d s
$$

We begin with an initial arbitrary approximation:

$$
\mathrm{u}_{0}(\mathrm{t}, \mathrm{x})=\mathrm{u}(0, x)=\frac{x}{2 \varepsilon}
$$

and using the iteration formula (6.4), we obtain the following successive approximations

$$
\begin{aligned}
\mathrm{u}_{1}(\mathrm{t}, \mathrm{x})= & \frac{\mathrm{x}}{2 \varepsilon}+\frac{x}{4 \varepsilon} \mathrm{t}+2 \mathrm{kt}, \\
\mathrm{u}_{2}(\mathrm{t}, \mathrm{x})= & \frac{x}{2 \varepsilon}+\frac{x}{4 \varepsilon} \mathrm{t}+2 \mathrm{k} t+\frac{x}{8 \varepsilon} \mathrm{t}^{2}+\frac{\mathrm{k}}{2} \mathrm{t}^{2}+\frac{\mathrm{k}}{4} \mathrm{t}^{3}+\frac{x}{48 \varepsilon} \mathrm{t}^{3}+\frac{\mathrm{k}}{2} \mathrm{t}+\frac{\mathrm{k}}{6} \mathrm{t}^{3}, \\
\mathrm{u}_{3}(\mathrm{t}, \mathrm{x})= & \frac{x}{2 \varepsilon}+\frac{x}{4 \varepsilon} \mathrm{t}+2 \mathrm{kt}+\frac{x}{8 \varepsilon} \mathrm{t}^{2}+\frac{\mathrm{k}}{2} \mathrm{t}^{2}+\frac{\mathrm{k}}{4} \mathrm{t}^{3}+\frac{11 \mathrm{k}}{96} \mathrm{t}^{4} \\
& +\frac{x}{16 \varepsilon} \mathrm{t}^{3}+\frac{x}{48 \varepsilon} \mathrm{t}^{4}+\frac{x}{192 \varepsilon} \mathrm{t}^{5}+\frac{x}{1152 \varepsilon} \mathrm{t}^{6}+\frac{x}{16128 \varepsilon} \mathrm{t}^{7}+\frac{7 \mathrm{k}}{240} \mathrm{t}^{5}+\frac{\mathrm{k}}{192} \mathrm{t}^{6}+\frac{\mathrm{k}}{2016} \mathrm{t}^{7} .
\end{aligned}
$$

This procedure can, in principle, be continued as far as desired, and the approximation will converge to its exact solution

$$
u(t, x)=\left(k t^{2}-4 k t-\frac{x}{\varepsilon}\right) /(t-2) .
$$

Since $P(t, x, \varepsilon, G)>0$, for $t \in] 0,2[\cup] 4, \infty\left[, \quad \frac{\partial P}{\partial G}>0, \quad \frac{\partial G}{\partial k}=\left(t^{2}-4 t\right) /(t-2)>0, \quad \frac{\partial F}{\partial k}>0, \quad \frac{\partial P}{\partial \varepsilon}<0, \quad \frac{\partial G}{\partial \varepsilon}=\right.$ $x / \varepsilon^{2}(t-2)<0$ for $\left.t \in\right] 0,2\left[\right.$. That is, $(\bar{Z})_{t}-\bar{\varepsilon} \bar{Z}(\bar{Z})_{x}=2 \bar{K}$, a fuzzy number.

So Lemma 5.2 implies the result that $\bar{Z}(t, x)$ is a BFS see Figs. 3, 5, and Table 2. We easily see that

$$
z_{\mathfrak{i}}(0, x, \alpha)=\frac{\chi}{2 \varepsilon_{\mathfrak{i}}(\alpha)}
$$

for $i=1,2$, so $\bar{Z}(t, x)$ also satisfies the initial condition. The BFS that satisfies the initial condition may be written as

$$
\bar{Z}(t, x)=\left(\bar{K} t^{2}-4 \bar{K} t-\frac{x}{\bar{\varepsilon}}\right) /(t-2)
$$

for all $t \in] 0,2[, x \in[0,1]$.

$\frac{\partial P}{\partial \varepsilon}<0, \quad \frac{\partial G}{\partial \varepsilon}>0$ for $\left.t \in\right] 4, \infty[$ or $P(t, x, \varepsilon, G)<0$, for $t \in] 2,4[$, then there is no BFS Lemma 5.2, see Fig. 4 and Table 3 . We proceed to look for a SS. We must solve for $t \in] 4, \infty[$,

$$
\begin{aligned}
& \left(u_{1}(t, x, \alpha)\right)_{t}-\varepsilon_{2}(\alpha) u_{1}(t, x, \alpha)\left(u_{1}(t, x, \alpha)\right)_{x}=2 k_{1}(\alpha), \\
& \left(u_{2}(t, x, \alpha)\right)_{t}-\varepsilon_{1}(\alpha) u_{2}(t, x, \alpha)\left(u_{2}(t, x, \alpha)\right)_{x}=2 k_{2}(\alpha),
\end{aligned}
$$

subject to $u_{\mathfrak{i}}(0, x, \alpha)=c_{\mathfrak{i}}(\alpha) x / 2 \varepsilon_{\mathfrak{i}}(\alpha)$ for $i=1,2$ and

$$
\overline{\mathrm{K}}[\alpha]=\left[\mathrm{k}_{1}(\alpha), \mathrm{k}_{2}(\alpha)\right] \quad \text { and } \quad \bar{\varepsilon}[\alpha]=\left[\varepsilon_{1}(\alpha), \varepsilon_{2}(\alpha)\right] .
$$

By VIM, the solution is

$$
u_{1}(t, x, \alpha)=\left(k_{1}(\alpha) t^{2}-4 k_{1}(\alpha) t-\frac{x}{\varepsilon_{2}(\alpha)}\right) /(t-2), \quad u_{2}(t, x, \alpha)=\left(k_{2}(\alpha) t^{2}-4 k_{2}(\alpha) t-\frac{x}{\varepsilon_{1}(\alpha)}\right) /(t-2) .
$$

Now we show $\left[u_{1}(t, x, \alpha), u_{2}(t, x, \alpha)\right]$ defines $\alpha$-cut of a fuzzy number. 
Thus we only need to check if $\frac{\partial u_{1}}{\partial \alpha}>0$ and $\frac{\partial u_{2}}{\partial \alpha}<0$. Since $u_{i}(t, x, \alpha)$ are continuous and $u_{1}(t, x, 1)=$ $\mathfrak{u}_{2}(t, x, 1)$, there is a region $\mathfrak{R}$ contained in $] 4, \infty[\times[0,1]$ for which the SS exists and in $] 4, \infty[\times[0,1]-\mathfrak{R}$ there may be no SS.

Since $\bar{K}$ and $\bar{\varepsilon}$ are triangular fuzzy numbers, hence, we pick simple fuzzy parameter so that $k_{1}^{\prime}(\alpha)=$ $\varepsilon_{1}^{\prime}(\alpha)=\lambda$ and $k_{2}^{\prime}(\alpha)=\varepsilon_{2}^{\prime}(\alpha)=-\lambda$. Then, for the SS we need

$$
\frac{\partial u_{1}}{\partial \alpha}=\lambda\left(t^{2}-4 t-x /\left(\varepsilon_{2}\right)^{2}\right) /(t-2)>0, \quad \frac{\partial u_{2}}{\partial \alpha}=-\lambda\left(t^{2}-4 t-x /\left(\varepsilon_{1}\right)^{2}\right) /(t-2)<0 .
$$

Therefore inequalities hold if

$$
t^{2}-4 t-x /\left(\varepsilon_{1}\right)^{2}>0
$$

for $t \in] 4, \infty[, x \in[0,1]$. The inequality (6.5) holds if

$$
0 \leqslant x \leqslant 1, \quad t \geqslant 2+\sqrt{4+\frac{x}{\left(\varepsilon_{1}\right)^{2}}} \text { for all } \alpha \in[0,1] .
$$

So under the above assumptions we may choose

$$
\mathfrak{R}=\left\{(t, x) \mid 0 \leqslant x \leqslant 1, \quad t \geqslant 2+\sqrt{4+\frac{x}{\left(\varepsilon_{1}\right)^{2}}} \text { for all } \alpha \in[0,1]\right\},
$$

and the SS exists on $\mathfrak{R}$ in form Eqs (6.6) see Fig. 4.

$$
\overline{\mathrm{U}}(\mathrm{t}, \mathrm{x})=\left(\overline{\mathrm{K}} \mathrm{t}^{2}-4 \overline{\mathrm{K}} \mathrm{t}-\frac{\mathrm{x}}{\overline{\bar{\varepsilon}}}\right) /(\mathrm{t}-2)
$$

for all $t \in] 4, \infty[, x \in[0,1]$.

Table 2: Comparison of the solution $u(t, x)$ with $u_{1}(t, x, \alpha)$ and $u_{2}(t, x, \alpha)$ for $\alpha=0 t \in[0,2[, \bar{\varepsilon}=\overline{-1}$.

\begin{tabular}{|c|c|c|c|c|c|c|c|c|c|}
\hline & \multicolumn{3}{|c|}{$u(t, x)$} & \multicolumn{3}{c|}{$\mathfrak{u}_{1}(t, x, \alpha)$} & \multicolumn{3}{c|}{$\mathfrak{u}_{2}(t, x, \alpha)$} \\
\hline$t \backslash x$ & 0.0495 & 0.1980 & 0.3465 & 0.0495 & 0.1980 & 0.3465 & 0.0495 & 0.1980 & 0.3465 \\
\hline 0 & 0.2005 & 0.8356 & 1.5314 & 0.1003 & 0.4178 & 0.7657 & 0.3008 & 1.2534 & 2.2971 \\
0.0099 & 0.1980 & 0.8329 & 1.5284 & 0.0986 & 0.4160 & 0.7637 & 0.2957 & 1.2479 & 2.2911 \\
0.0198 & 0.1955 & 0.8301 & 1.5254 & 0.0969 & 0.4141 & 0.7617 & 0.2906 & 1.2424 & 2.2851 \\
\hline
\end{tabular}

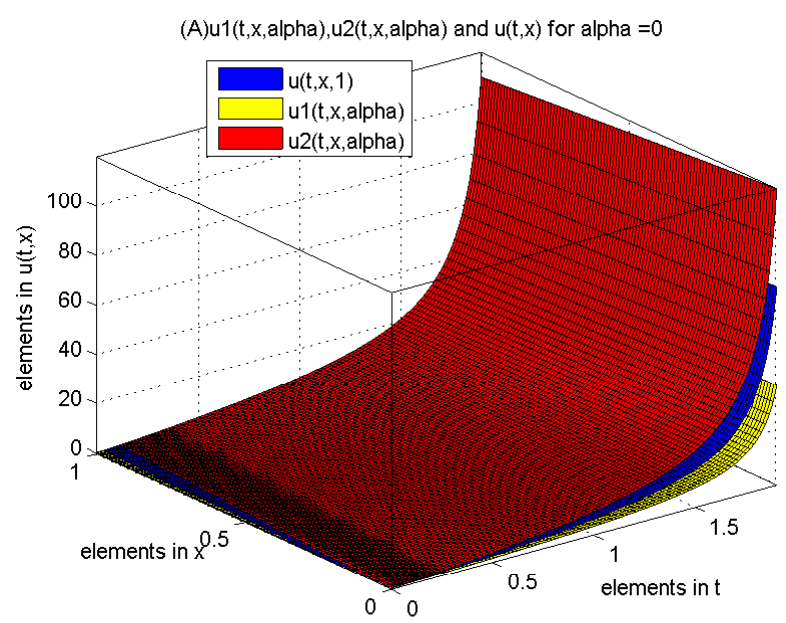

Figure 3: The surfaces shows the fuzzy solution for $\alpha=0$ for (6.3), when $\bar{k}=\overline{2}$ and $\bar{\varepsilon}=\overline{-1}$. 
Table 3: Comparison of the solution $u(t, x)$ with $u_{1}(t, x, \alpha)$ and $u_{2}(t, x, \alpha)$ for $\left.\left.\alpha=0 t \in\right] 2,5\right], \bar{\varepsilon}=\overline{-1}$.

\begin{tabular}{|c|c|c|c|c|c|c|c|c|c|}
\hline & \multicolumn{3}{|c|}{$u(t, x)$} & \multicolumn{3}{c|}{$\mathfrak{u}_{1}(t, x, \alpha)$} & \multicolumn{3}{c|}{$\mathfrak{u}_{2}(t, x, \alpha)$} \\
\hline$t \backslash x$ & 0.0495 & 0.1980 & 0.3465 & 0.0495 & 0.1980 & 0.3465 & 0.0495 & 0.1980 & 0.3465 \\
\hline 2.0297 & -264.04 & -100.59 & -61.70 & -134.08 & -50.21 & -30.79 & -402.24 & -150.63 & -92.46 \\
2.0792 & -265.94 & -99.59 & -61.12 & -132.41 & -49.58 & -30.43 & -397.24 & -148.76 & -91.30 \\
2.1287 & -263.44 & -98.65 & -60.55 & -130.74 & -48.96 & -30.05 & -392.24 & -146.88 & -90.15 \\
\hline
\end{tabular}

(B) $u 1(t, x, a l p h a), u 2(t, x, a l p h a)$ and $u(t, x)$ for alpha $=0$

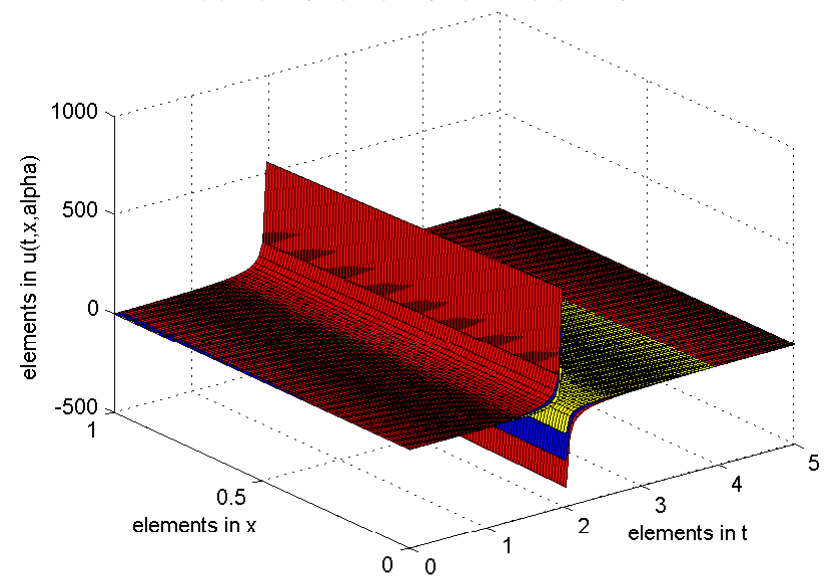

Figure 4: The surfaces shows the fuzzy solution for $\alpha=0, t \in[0,5] \backslash\{2\}$ for $(6.3)$, when $\bar{k}=\overline{2}$ and $\bar{\varepsilon}=\overline{-1}$.

(C) $\mathrm{u} 1(\mathrm{t}, \mathrm{x}, \mathrm{alpha}), \mathrm{u} 2(\mathrm{t}, \mathrm{x}, \mathrm{alpha})$ and $\mathrm{u}(\mathrm{t}, \mathrm{x})$ for alpha in $[0,1]$

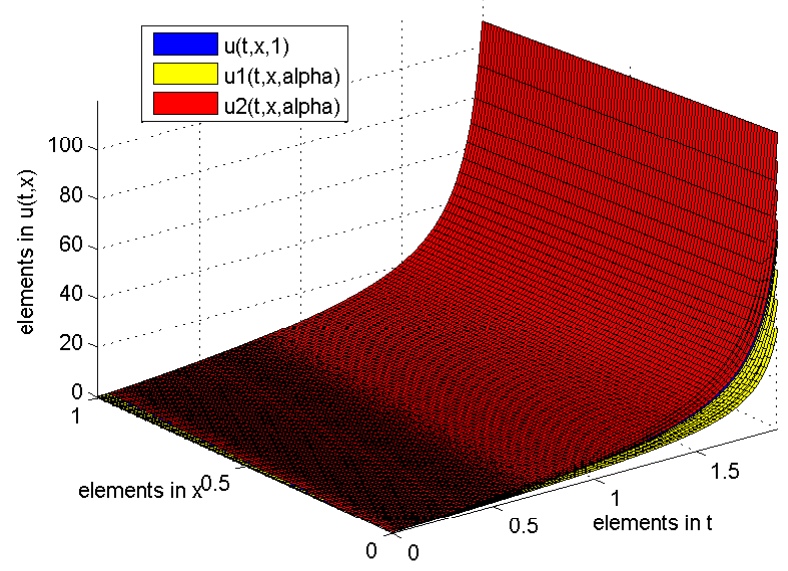

Figure 5: The surfaces shows the fuzzy solution for $\alpha \in[0,1]$ for $(6.3)$, when $\bar{k}=\overline{2}$ and $\bar{\varepsilon}=\overline{-1}$.

\section{Conclusion}

The proposed strategy in this paper may be useful the coefficients, initial values and forcing terms are fuzzy, for solving burgers equation. This strategy consists of two types of solutions: Buckley-Feuring solution and Seikkala solution, using the VIM. And has been successfully applied to new theorem. we proved in the first if BFsolution exists because if it does it is the same as the S-solution as shown in Fig. 1 (for $\alpha=0)$, Fig. $2(\forall \alpha \in[0,1])$. However, if the BF-solution fails to exist as shown in Fig. 3 (for $\alpha=0, t \in] 2,5], x \in[0,1]$ ) we check if the S-solution exists as shown in Fig. 4 (for $\alpha=0$ ) and Fig. 5 $(\forall \alpha \in[0,1]$, and $\mathrm{t} \in[0,2[, x \in[0,1])$. When the S-solution fails to exist we offer no solution to the fuzzy 
burger's equation.

For further research we propose the study for fuzzy Burgers equations, by the using the conformable differentiability concept [11, 12]. In addition, we propose to extend the results of the present paper and to combine them with the results in [10] for Hybrid fuzzy differential equations.

\section{References}

[1] T. A. Abassy, M. A. El-Tawil, H. El Zoheiry, Toward a modified variational iteration method, J. Comput. Appl. Math., 207 (2007), 137-147. 3, 6

[2] N. Bildik, A. Konuralp, The Use of Variational Iteration Method, Differential Transform Method and Adomian Decomposition Method for Solving Different Types of Nonlinear Partial Differential Equations, Int. J. Nonlinear Sci. Numer. Simul., 7 (2006), 65-70. 6

[3] J. J. Buckley, T. Feuring, Fuzzy differential equations, Fuzzy Sets and Systems, 110 (2000), 43-54. 5

[4] J. J. Buckley, Y. X. Qu, Solving fuzzy equations: a new solution concept, Fuzzy Sets and Systems, 39 (1991), $291-301$. 1,5

[5] L. S. Chadli, A. Harir, S. Melliani, Solutions of fuzzy wave-like equations by variational iteration method, Ann. Fuzzy Math. Inform., 8 (2014), 527-547. 1, 5

[6] P. Diamond, P. E. Kloeden, Metric Spaces of Fuzzy Sets: Theory and Applications, World Scientific Publishing Co., River Edge, (1994). 2.1, 2.2

[7] R. Getschel, W. Voxman, Elementary fuzzy calculus, Fuzzy sets and Systems, 18 (1986), 31-43. 2, 5

[8] A. Harir, S. Melliani, L. S. Chadli, Fuzzy Space-Time Fractional Telegraph Equations, Int. J. Math. Trends Tech., 64 (2018), 101-108.

[9] A. Harir, S. Melliani, L. S. Chadli, Existence and Uniqueness of a Fuzzy Solution for some Fuzzy Neutral partial Differential Equation with nonlocal condition, Int. J. Math. Trends Tech., 65 (2019), 102-108. 2

[10] A. Harir, S. Melliani, L. S. Chadli, Hybrid Fuzzy Differential Equations, AIMS Math., 5 (2019), 273-285. 7

[11] A. Harir, S. Melliani, L. S. Chadli, Fuzzy generalized conformable fractional derivative. Advances in Fuzzy Systems, Adv. Fuzzy Syst., 2020 (2020), 7 pages. 7

[12] A. Harir, S. Melliani, L. S. Chadli, E. Minchev, Solutions of fuzzy fractional heat-like and wave-like equations by variational iteration method, Int. J. Contemp. Math. Sci., 15 (2020), 11-35. 7

[13] J.-H. He, A variational iteration approach to nonlinear problems and its applications, Mech. Appl., 20 (1998), 30-31. 1

[14] J.-H. He, Variational principles for some nonlinear partial differential equations with variable coefficients, Chaos Solitons Fractals, 19 (2004), 847-851. 4

[15] J.-H. He, Some asymptotic methods for strongly nonlinear equations, Internat. J. Modern Phys. B, 20 (2006), 1141-1199. 1,4

[16] M. Ma, M. Friedman, A. Kandel, A new fuzzy arithmetic, Fuzzy Sets and Systems, 108 (1999), 83-90. 2.2

[17] S. Melliani, A. Harir, L. S. Chadli, Solutions of fuzzy heat-like equations by variational iteration method, Ann. Fuzzy Math. Inform., 10 (2015), 29-44. 1, 5

[18] S. Seikkala, On the fuzzy initial value problem, Fuzzy Sets and Systems, 24 (1987), 319-330. 1, 5

[19] K. Shah, A. Ullah, Using a hybrid technique for analytical solution of coupled system of two-dimensional Burger's equations, Results in Nonlinear Anal., 1 (2018), 107-115. 6

[20] A.-M. Wazwaz, The variational iteration method for solving linear and nonlinear systems of PDEs, Comput. Math. Appl., 54 (2007), 895-902. 4, 4, 4

[21] A.-M. Wazwaz, A. Gorguis, Exact solutions for heat-like and wave-like equations with variable coefficients, Appl. Math. Comput., 149 (2004), 15-29. 4

[22] L. A. Zadeh, Fuzzy sets, Inf. Control, 8 (1965), 338-353. 1 